\title{
3 Research Square

\section{U-Shaped Association Between Serum Bilirubin Levels and Peripheral Arterial Disease in Chinese Males with Hypertension}

\section{Yumeng Shi}

Nanchang University Second Affiliated Hospital

\section{Lihua Hu}

Nanchang University Second Affiliated Hospital

Minghui Li

Nanchang University Second Affiliated Hospital

Xiao Huang

Nanchang University Second Affiliated Hospital

Congcong Ding

Nanchang University Second Affiliated Hospital

Wei Zhou

Nanchang University Second Affiliated Hospital

\section{Tao Wang}

Nanchang University Second Affiliated Hospital

Lin juan Zhu

Nanchang University Second Affiliated Hospital

Huihui Bao

Nanchang University Second Affiliated Hospital

Xiaoshu Cheng ( $\square$ xiaoshumenfan126@163.com)

the second affiliated hospital of nanchang university https://orcid.org/0000-0001-7445-1988

\section{Research article}

Keywords: : total bilirubin, ankle-brachial index, peripheral arterial disease, hypertension, U-shaped curve, males

Posted Date: July 8th, 2020

DOI: https://doi.org/10.21203/rs.3.rs-40589/v1

License: (c) (1) This work is licensed under a Creative Commons Attribution 4.0 International License.

Read Full License 


\section{Abstract}

Background. Previous studies indicated that serum total bilirubin might play an important role in peripheral artery disease (PAD). However, the effects of different levels of serum total bilirubin (TBiL) on PAD development remain an uncertainty and had limited data in male participants. We aimed to examine the prevalence of PAD and TBiL among Chinese male adults with hypertension.

Methods: A total of 5129 hypertension male subjects aged 27-93 years was included in the current study. The outcome was a peripheral arterial disease (PAD), defined as present when $A B I$ of either sides was $\leq$ 0.90 .

Results. Of 5,129 hypertension male participants, 194 (3.78\%) had PAD, and the median serum concentrations of TBiL were $14.10 \mu \mathrm{mol} / \mathrm{L}$ (interquartile range 11.00 to $18.60 \mu \mathrm{mol} / \mathrm{L}$ ). Compared to participants in Q2-Q3 of LgTBiL, there was a significantly increased risk of PAD for participants in both Q1 (OR,1.49; 95\% Cl: 1.04-2.14) and Q4 (OR,1.70; 95\%Cl: 1.16-2.48). After adjusting for potential confounders, a nonlinear U-shaped relationship was detected between TBiL and PAD, and calculated the inflection point was $1.08(\mathrm{LgTBiL}=1.08, \mathrm{TBiL}=12.02 \mu \mathrm{mol} / \mathrm{L})$. The ORs $(95 \% \mathrm{Cls})$ were $0.11(0.02,0.83)$ on the left side of an inflection point and $5.26(1.59,17.38)$ on the right side of the inflection point, respectively. Similar results were found in various subgroups.

Conclusions: This cross-sectional study had shown that there was a U-shaped curve for the risk of PAD with TBiL concentration in Chinese males with hypertension, with a turning point at $12.02 \mu \mathrm{mol} / \mathrm{L}$.

\section{Introduction}

As a prevalent cardiovascular disease (CVD), peripheral arterial disease (PAD) had a high fatality rate(1), and in recent years, the incidence rate of PAD has increased year by year(2). Hypertension is one of the significant risk factors for PAD; the number of patients with hypertension is estimated at 245 million in China(3). Several studies have shown that increases in blood pressure are strictly related to increased PAD risk(4-6). Therefore, there is an urgent need to identify novel modifiable risk factors to inform PAD prevention in the hypertensive population. The ankle-brachial index (ABI) is a noninvasive method for diagnosis and detection of PAD and is widely used for risk assessment of atherosclerosis and cardiovascular diseases(7), and PAD was defined as an ABI of $\leq 0.90$ in either leg(8).

The effects of serum total bilirubin (TBiL) on the risk of CVD have received considerable attention(9-12). Bilirubin is a potent antioxidant under physiological conditions; a higher TBiL level could be a protector factor for atherosclerosis(13). At the same time, some studies have shown that elevated TBiL levels were negatively correlated with coronary artery disease (CAD) (14-16), arterial stiffness(12), and PAD(17-19). Excessive TBiL is probably an indicator of the potential liver cell damage associated with an increased risk of CVD(20). Nevertheless, the possible effect of excessive TBiL on the risk of increased PAD has not been examined in previous studies. Moreover, androgens in men inhibit bilirubin excretion, and estrogen 
in women increases their excretion(21). Compared with females, males have more risk factors for PAD and higher bilirubin levels.

Therefore, this study aimed to assess the relationship between the TBiL and PAD in Chinese hypertensive male subjects to address this gap, as mentioned earlier in knowledge.

\section{Methods}

\section{Study Design and population}

All patients gave written informed consent. The Ethics Committees of the Institute of Biomedicine, Anhui Medical University, approved study protocols. In the current study, we included rural subjects from ongoing China H-type hypertension Registry Study (Registration number: ChiCTR1800017274). China Htype hypertension Registry Study is a real-world, multicenter, observational study, conducted from Wuyuan, Jiangxi province of China, beginning in July 2018. The aim of this study was to confirm that establish a national registry of patients with hypertension, investigate the prevalence and treatment of hypertension in China, and assess the related factors affecting its prognosis. Details regarding the inclusion and exclusion criteria of the study have been described elsewhere(22).

A total of 14,268 participants completed the investigation. Subject were excluded if they: 1 .were not hypertension $(n=34) ; 2$. had missed $A B I(n=3328)$ and TBiL value $(n=6) ; 3$. were females $(n=5771)$. The final analysis included 5,129 participants (Figure S1).

\section{Data collection}

Baseline information on sociodemographic characteristics, lifestyle habits, comorbidities, and medication use was obtained in-person interviews by well-trained research staff according to a standard protocol. Anthropometric parameters indicators included weight, height, waist circumference (WC), hip circumference (HC), systolic blood pressure (SBP), diastolic blood pressure (DBP), pulse, and ABI measurements were collected. BMI was calculated as the body weight in kilograms divided by the square of the height in meters $\left(\mathrm{kg} / \mathrm{m}^{2}\right)$.

After an overnight fast of $12-15 \mathrm{~h}$, blood samples were collected utilizing venipuncture and were immediately frozen and stored $-80^{\circ} \mathrm{C}$ until analysis. The measured variables included serum total bilirubin (TBiL), serum homocysteine (Hcy), serum creatinine, lipids, and fasting blood glucose (FBG). The formula for estimated glomerular filtration rate (eGFR) used the Chronic Kidney Disease Epidemiology Collaboration (CKD-EPI) Eq. (23). All of these parameters were measured using automatic clinical analyzers (Beckman Coulter) at the core laboratory of the National Clinical Research Center for Kidney Disease, Guangzhou, China. All laboratory measurements met a standardization and certification program.

\section{Ankle-Brachial Index Measurement}


The Ankle-Brachial Index ( $\mathrm{ABI})$ was automatically measured with the subject in the supine position after having rested for more than 10 minutes, using Omron Colin BP-203RPE III device (Omron Health Care, Kyoto, Japan) and calculated for each leg by dividing the SBP obtained at the ankle level in the respective leg by the SBP of the brachial artery(24). The lowest value of the ABI was used in the analysis. PAD was defined as an $A B I$ of $\leq 0.90$ in either leg(8).

\section{Other Definitions}

Hypertension was defined as SBP $\geq 140 \mathrm{~mm} \mathrm{Hg}$ and/or DBP $\geq 90 \mathrm{~mm} \mathrm{Hg}$, and also if the individual was on antihypertensive medication within 2 weeks(25). Diabetes mellitus was defined as a self-reported physician diagnosis of diabetes or FBG concentration of $\geq 7.0 \mathrm{mmol} / \mathrm{L}$ or the use of glucose-lowering drugs. Chronic kidney disease (CKD) was defined as eGFR $<60 \mathrm{~mL} / \mathrm{min} / 1.73 \mathrm{~m}^{2}$ or a self-reported physician diagnosis of CKD.

\section{Covariates}

These selected covariates are closely related to PAD. Continuous variables included age, systolic blood pressure (SBP), diastolic blood pressure (DBP), pulse, BMI, HC, WC, laboratory results [FBG, Hcy, alanine aminotransferase(ALT), aspartate aminotransferase(AST), total cholesterol (TC), triglycerides (TG), highdensity lipoprotein cholesterol (HDL-C), estimated glomerular filtration rate (eGFR)] Categorical variables consisted of smoking status (never, former or current), drinking status (never, former or current), coronary heart disease (no or yes), and medication use (antihypertensive drugs, glucose-lowering drugs, lipidlowering drugs).

\section{Statistical Analysis}

We divided the study population into four groups based on participants by quartiles of LgTBiL levels. Baseline characteristics presented as mean, median (SD or interquartile range) for continuous variables and count (percentage) for categorical variables. Differences in population characteristics were compared using Student's t-test, chi-square test, or Wilcoxon signed-rank test. The distribution of serum concentrations of TBiL was strongly skewed toward the left. Thus, we performed the Log10 transformation (LgTBiL) before analysis. We constructed three models to examine the association between LgTBiL and the prevalence of PAD. The crude model was adjusted for none; model 1 was adjusted for age, BMI, WC, HC, SBP, DBP, pulse, smoking status, alcohol consumption, and model 2 was adjusted for all variables in Model 1 plus adjusted for FBG, TC, TG, Hcy, HDL-C, AST, ALT, eGFR, CHD, antihypertensive drugs, glucose-lowering drugs, lipid-lowering drugs. Multivariate logistic regression was used to assess the odds ratios (ORs) and 95\% confidence intervals (CI) for PAD in each LgTBiL tertile. To characterize the shape of the relationship between LgTBiL and PAD prevalence, a generalized additive model, and a smooth curve fitting (penalized spline method) was performed. If nonlinearity was detected, we first use a recursive algorithm to calculate the inflection points and then construct a two-segment binary logistic model on both sides of the inflection points. As additional exploratory analyses, possible modifications of the LgTBiL effects on the prevalence of PAD in participants separated by the turning point of LgTBiL were also assessed for variables including age (<65 vs. $\geq 65$ years), BMI (<24 vs. 
$\geq 24 \mathrm{~kg} / \mathrm{m}^{2}$ ), smoking status (never vs. Former vs. current), AST ( $\leq 40 \mathrm{vs.} \geq 40 \mathrm{U} / \mathrm{L}$ ), diabetes mellitus (no vs. yes), CKD (no vs. yes). A 2-tailed $P<0.05$ was considered to be statistically significant in all analyses.The Empower (R; www.empowerstats.com; X\&Y Solutions, Inc, Boston, MA, USA) and the statistical package R (http://www.R-project.org, The R Foundation) were used for all data analyses.

\section{Results}

\section{Study participants and baseline characteristics}

As shown in the flow chart (Figure S1), a total of 5,129 hypertension male subjects were included in the current study. The mean age of our study participants was $63.92 \pm 9.62$ years, the median serum concentrations of TBiL was $14.10 \mu \mathrm{mol} / \mathrm{L}$ (interquartile range 11.00 to $18.60 \mu \mathrm{mol} / \mathrm{L}$ ); $194(3.78 \%$ ) had PAD, and 828 (16.14\%) had diabetes.

Baseline characteristics of study participants stratified by quartiles of LgTBiL levels are summarized in Table 1. Participants with higher levels of LgTBiL tended to be a current alcohol drinker, and had higher rates of diabetes mellitus; and higher value in DBP, pulse, FBG, BMI, WC, HC, TC, HDL, AST, ALT, eGFR, albumin; had lower rates of PAD, CKD and smokers; and lower value in age (all $P<0.01$ ). There were no statistically significant between the four groups in terms of SBP, Hcy, TG, stroke, CHD, antihypertensive drugs, glucose-lowering drugs, or lipid-lowering drugs (all $P>0.05$ ). 
Table 1

Baseline characteristics of study participants by quartiles of baseline LgTBiL levels

\begin{tabular}{|c|c|c|c|c|c|c|}
\hline \multirow[t]{2}{*}{ Characteristics } & \multirow[t]{2}{*}{ Total } & \multicolumn{4}{|c|}{ LgTBiL, $\mu \mathrm{mol} / \mathrm{L}$} & \multirow{2}{*}{$\begin{array}{l}P \\
\text { value }\end{array}$} \\
\hline & & $\begin{array}{l}\text { Q1 (< } \\
1.04)\end{array}$ & $\begin{array}{l}\text { Q2 (1.04- } \\
1.15)\end{array}$ & $\begin{array}{l}\text { Q3 (1.15- } \\
1.27)\end{array}$ & Q4 ( $\geq 1.27)$ & \\
\hline$N$ & 5129 & 1281 & 1272 & 1293 & 1283 & \\
\hline Age,year & $\begin{array}{l}63.92 \pm \\
9.62\end{array}$ & $\begin{array}{l}64.37 \pm \\
9.23\end{array}$ & $64.23 \pm 9.44$ & $\begin{array}{l}63.60 \pm \\
9.86\end{array}$ & $63.50 \pm 9.91$ & 0.043 \\
\hline $\mathrm{BMI}, \mathrm{kg} / \mathrm{m} 2$ & $\begin{array}{l}23.36 \pm \\
4.01\end{array}$ & $\begin{array}{l}22.98 \pm \\
3.44\end{array}$ & $23.43 \pm 3.60$ & $\begin{array}{l}23.44 \pm \\
3.46\end{array}$ & $23.58 \pm 5.23$ & 0.001 \\
\hline WC & $\begin{array}{l}83.85 \pm \\
9.69\end{array}$ & $\begin{array}{l}82.79 \pm \\
9.88\end{array}$ & $84.14 \pm 9.72$ & $\begin{array}{l}84.08 \pm \\
9.36\end{array}$ & $84.40 \pm 9.71$ & $\begin{array}{l}< \\
0.001\end{array}$ \\
\hline $\mathrm{HC}$ & $\begin{array}{l}91.83 \pm \\
6.82\end{array}$ & $\begin{array}{l}91.31 \pm \\
6.79\end{array}$ & $92.02 \pm 7.20$ & $\begin{array}{l}92.03 \pm \\
6.71\end{array}$ & $91.98 \pm 6.53$ & 0.016 \\
\hline
\end{tabular}

Smoking status,

$\mathrm{N}(\%)$

$\begin{array}{llllll}\text { Never } & 1070 & 213 & 241 & 292 & 324 \\ & (20.86 \%) & (16.63 \%) & (18.95 \%) & (22.58 \%) & (25.25 \%) \\ \text { Former } & 1516 & 358 & 358 & 395 & 405 \\ & (29.56 \%) & (27.95 \%) & (28.14 \%) & (30.55 \%) & (31.57 \%) \\ \text { Current } & 2543 & 710 & 673 & 606 & 554 \\ & (49.58 \%) & (55.43 \%) & (52.91 \%) & (46.87 \%) & (43.18 \%)\end{array}$

Drinking status,

$\mathrm{N}(\%)$

$\begin{array}{llllll}\text { Never } & \begin{array}{l}1906 \\ \text { (37.17\%) }\end{array} & \begin{array}{l}546 \\ (42.62 \%)\end{array} & \begin{array}{l}470 \\ (36.98 \%)\end{array} & \begin{array}{l}461 \\ (35.65 \%)\end{array} & \begin{array}{l}429 \\ (33.44 \%)\end{array} \\ \text { Former } & 1090 & 289 & 294 & 290 & 217 \\ & (21.26 \%) & (22.56 \%) & (23.13 \%) & (22.43 \%) & (16.91 \%) \\ \text { Current } & 2132 & 446 & 507 & 542 & 637 \\ & (41.58 \%) & (34.82 \%) & (39.89 \%) & (41.92 \%) & (49.65 \%)\end{array}$

Abbreviation: PAD, peripheral arterial disease; $B M I$, body mass index; WC, waist circumference; $\mathrm{HC}$, hip circumference; SBP, systolic blood pressure; DBP, diastolic blood pressure; CHD, coronary heart disease; CKD, chronic kidney disease; FBG: fasting blood glucose; Hcy, homocysteine; eGFR, estimated glomerular filtration rate; HDL-C, high-density lipoprotein cholesterol; TC, total cholesterol; TG, Triglycerides; TBiL, total bilirubin; ALT, alanine aminotransferase; AST, aspartate aminotransferase; eGFR, estimated glomerular filtration rate.

\$diabetes mellitus was defined as self-reported physician diagnosis of diabetes or FBG concentration $\geq 7.0 \mathrm{mmol} / \mathrm{L}$ or use of glucose-lowering drugs.

\#CKD was defined as eGFR $<60 \mathrm{~mL} / \mathrm{min} / 1.73 \mathrm{~m}^{2}$ or a self-reported physician diagnosis of CKD. 


\begin{tabular}{|c|c|c|c|c|c|c|}
\hline \multirow[t]{2}{*}{ Characteristics } & \multirow[t]{2}{*}{ Total } & \multicolumn{4}{|c|}{ LgTBiL, $\mu \mathrm{mol} / \mathrm{L}$} & \multirow{2}{*}{$\begin{array}{l}P \\
\text { value }\end{array}$} \\
\hline & & $\begin{array}{l}\text { Q1 (< } \\
1.04)\end{array}$ & $\begin{array}{l}\text { Q2 (1.04- } \\
1.15)\end{array}$ & $\begin{array}{l}\text { Q3 (1.15- } \\
1.27)\end{array}$ & $\mathrm{Q} 4(\geq 1.27)$ & \\
\hline SBP, mmHg & $\begin{array}{l}146.63 \pm \\
17.96\end{array}$ & $\begin{array}{l}147.16 \pm \\
18.60\end{array}$ & $\begin{array}{l}146.68 \pm \\
17.37\end{array}$ & $\begin{array}{l}146.04 \pm \\
17.32\end{array}$ & $\begin{array}{l}146.65 \pm \\
18.52\end{array}$ & 0.475 \\
\hline DBP, mmHg & $\begin{array}{l}90.35 \pm \\
11.06\end{array}$ & $\begin{array}{l}89.30 \pm \\
11.22\end{array}$ & $\begin{array}{l}90.06 \pm \\
10.93\end{array}$ & $\begin{array}{l}90.48 \pm \\
11.12\end{array}$ & $\begin{array}{l}91.55 \pm \\
10.85\end{array}$ & $\begin{array}{l}< \\
0.001\end{array}$ \\
\hline pulse, bpm & $\begin{array}{l}74.41 \pm \\
14.06\end{array}$ & $\begin{array}{l}73.28 \pm \\
13.84\end{array}$ & $\begin{array}{l}73.73 \pm \\
13.11\end{array}$ & $\begin{array}{l}74.57 \pm \\
13.53\end{array}$ & $\begin{array}{l}76.06 \pm \\
15.51\end{array}$ & $\begin{array}{l}<.001 \\
0.001\end{array}$ \\
\hline \multicolumn{7}{|l|}{ Laboratory data } \\
\hline Hcy, $\mu \mathrm{mol} / \mathrm{L}$ & $\begin{array}{l}20.50 \pm \\
13.64\end{array}$ & $\begin{array}{l}20.17 \pm \\
12.99\end{array}$ & $\begin{array}{l}19.94 \pm \\
12.67\end{array}$ & $\begin{array}{l}21.13 \pm \\
15.31\end{array}$ & $\begin{array}{l}20.77 \pm \\
13.42\end{array}$ & 0.103 \\
\hline $\mathrm{FBG}, \mathrm{mmol} / \mathrm{L}$ & $6.07 \pm 1.50$ & $\begin{array}{l}5.94 \pm \\
1.40\end{array}$ & $6.01 \pm 1.47$ & $6.12 \pm 1.52$ & $6.19 \pm 1.59$ & $\begin{array}{l}< \\
0.001\end{array}$ \\
\hline $\mathrm{TC}, \mathrm{mmol} / \mathrm{L}$ & $4.93 \pm 1.06$ & $\begin{array}{l}4.83 \pm \\
1.09\end{array}$ & $4.93 \pm 1.06$ & $4.97 \pm 1.05$ & $4.99 \pm 1.05$ & $\begin{array}{l}< \\
0.001\end{array}$ \\
\hline $\mathrm{TG}, \mathrm{mmol} / \mathrm{L}$ & $1.65 \pm 1.26$ & $\begin{array}{l}1.67 \pm \\
1.54\end{array}$ & $1.61 \pm 1.09$ & $1.65 \pm 1.15$ & $1.68 \pm 1.20$ & 0.570 \\
\hline $\mathrm{HDL}-\mathrm{C}, \mathrm{mmol} / \mathrm{L}$ & $1.55 \pm 0.44$ & $\begin{array}{l}1.47 \pm \\
0.41\end{array}$ & $1.53 \pm 0.43$ & $1.57 \pm 0.43$ & $1.63 \pm 0.49$ & $\begin{array}{l}< \\
0.001\end{array}$ \\
\hline AST, U/L & $\begin{array}{l}27.98 \pm \\
21.81\end{array}$ & $\begin{array}{l}26.26 \pm \\
16.53\end{array}$ & $\begin{array}{l}26.73 \pm \\
11.58\end{array}$ & $\begin{array}{l}27.44 \pm \\
10.84\end{array}$ & $\begin{array}{l}31.48 \pm \\
36.91\end{array}$ & $\begin{array}{l}< \\
0.001\end{array}$ \\
\hline ALT, U/L & $\begin{array}{l}22.25 \pm \\
20.96\end{array}$ & $\begin{array}{l}20.79 \pm \\
15.60\end{array}$ & $\begin{array}{l}21.27 \pm \\
13.95\end{array}$ & $\begin{array}{l}22.36 \pm \\
15.21\end{array}$ & $\begin{array}{l}24.55 \pm \\
32.85\end{array}$ & $\begin{array}{l}< \\
0.001\end{array}$ \\
\hline Albumin, g/L & $\begin{array}{l}46.49 \pm \\
4.16\end{array}$ & $\begin{array}{l}45.26 \pm \\
4.13\end{array}$ & $46.29 \pm 3.90$ & $\begin{array}{l}47.06 \pm \\
4.09\end{array}$ & $47.35 \pm 4.20$ & $\begin{array}{l}<.001 \\
0.001\end{array}$ \\
\hline $\begin{array}{l}\text { TBiL,median } \\
\text { (IQR), } \mu \mathrm{mol} / \mathrm{L}\end{array}$ & $\begin{array}{l}14.10 \\
(11.00- \\
18.60)\end{array}$ & $\begin{array}{l}9.20 \\
(7.90- \\
10.10)\end{array}$ & $\begin{array}{l}12.50 \\
(11.70- \\
13.30)\end{array}$ & $\begin{array}{l}16.00 \\
(15.00- \\
17.10)\end{array}$ & $\begin{array}{l}22.90 \\
(20.30- \\
27.60)\end{array}$ & $\begin{array}{l}< \\
0.001\end{array}$ \\
\hline
\end{tabular}

Abbreviation: PAD, peripheral arterial disease; BMI, body mass index; WC, waist circumference; $\mathrm{HC}$, hip circumference; SBP, systolic blood pressure; DBP, diastolic blood pressure; CHD, coronary heart disease; CKD, chronic kidney disease; FBG: fasting blood glucose; Hcy, homocysteine; eGFR, estimated glomerular filtration rate; HDL-C, high-density lipoprotein cholesterol; TC, total cholesterol; TG, Triglycerides; TBiL, total bilirubin; ALT, alanine aminotransferase; AST, aspartate aminotransferase; eGFR, estimated glomerular filtration rate.

\$diabetes mellitus was defined as self-reported physician diagnosis of diabetes or FBG concentration $\geq 7.0 \mathrm{mmol} / \mathrm{L}$ or use of glucose-lowering drugs.

${ }^{\#}$ CKD was defined as eGFR $<60 \mathrm{~mL} / \mathrm{min} / 1.73 \mathrm{~m}^{2}$ or a self-reported physician diagnosis of CKD. 


\begin{tabular}{|c|c|c|c|c|c|c|}
\hline \multirow[t]{2}{*}{ Characteristics } & \multirow[t]{2}{*}{ Total } & \multicolumn{4}{|c|}{ LgTBiL, $\mu \mathrm{mol} / \mathrm{L}$} & \multirow{2}{*}{$\begin{array}{l}P \\
\text { value }\end{array}$} \\
\hline & & $\begin{array}{l}\text { Q1 (< } \\
1.04)\end{array}$ & $\begin{array}{l}\text { Q2 (1.04- } \\
1.15)\end{array}$ & $\begin{array}{l}\text { Q3 (1.15- } \\
1.27)\end{array}$ & $\mathrm{Q} 4(\geq 1.27)$ & \\
\hline $\begin{array}{l}\text { eGFR, } \\
\mathrm{mL} / \mathrm{min} / 1.73 \mathrm{~m}^{2}\end{array}$ & $\begin{array}{l}86.18 \pm \\
20.46\end{array}$ & $\begin{array}{l}82.96 \pm \\
23.79\end{array}$ & $\begin{array}{l}86.68 \pm \\
19.40\end{array}$ & $\begin{array}{l}86.48 \pm \\
19.98\end{array}$ & $\begin{array}{l}88.61 \pm \\
17.83\end{array}$ & $\dot{0.001}$ \\
\hline \multicolumn{7}{|l|}{$\begin{array}{l}\text { Comorbidities, N } \\
\text { (\%) }\end{array}$} \\
\hline Stroke & $\begin{array}{l}403 \\
(7.86 \%)\end{array}$ & $\begin{array}{l}107 \\
(8.35 \%)\end{array}$ & $102(8.02 \%)$ & $\begin{array}{l}106 \\
(8.20 \%)\end{array}$ & $88(6.86 \%)$ & 0.484 \\
\hline $\mathrm{CHD}^{\#}$ & $\begin{array}{l}273 \\
(5.32 \%)\end{array}$ & $\begin{array}{l}55 \\
(4.29 \%)\end{array}$ & $72(5.66 \%)$ & 68 (5.26\%) & 78 (6.08\%) & 0.217 \\
\hline $\begin{array}{l}\text { Diabetes } \\
\text { mellitus } \$\end{array}$ & $\begin{array}{l}828 \\
(16.14 \%)\end{array}$ & $\begin{array}{l}188 \\
(14.68 \%)\end{array}$ & $\begin{array}{l}184 \\
(14.47 \%)\end{array}$ & $\begin{array}{l}219 \\
(16.94 \%)\end{array}$ & $\begin{array}{l}237 \\
(18.47 \%)\end{array}$ & 0.015 \\
\hline CKD & $\begin{array}{l}781 \\
(15.23 \%)\end{array}$ & $\begin{array}{l}263 \\
(20.53 \%)\end{array}$ & $\begin{array}{l}191 \\
(15.02 \%)\end{array}$ & $\begin{array}{l}182 \\
(14.08 \%)\end{array}$ & $\begin{array}{l}145 \\
(11.30 \%)\end{array}$ & $\begin{array}{l}< \\
0.001\end{array}$ \\
\hline PAD & $\begin{array}{l}194 \\
(3.78 \%)\end{array}$ & $\begin{array}{l}64 \\
(5.00 \%)\end{array}$ & $33(2.59 \%)$ & $45(3.48 \%)$ & $52(4.05 \%)$ & 0.013 \\
\hline \multicolumn{7}{|l|}{$\begin{array}{l}\text { Medication use, } \\
\text { N (\%) }\end{array}$} \\
\hline $\begin{array}{l}\text { Antihypertensive } \\
\text { drugs }\end{array}$ & $\begin{array}{l}3362 \\
(65.55 \%)\end{array}$ & $\begin{array}{l}844 \\
(65.89 \%)\end{array}$ & $\begin{array}{l}829 \\
(65.17 \%)\end{array}$ & $\begin{array}{l}849 \\
(65.66 \%)\end{array}$ & $\begin{array}{l}840 \\
(65.47 \%)\end{array}$ & 0.985 \\
\hline $\begin{array}{l}\text { Glucose- } \\
\text { lowering drugs }\end{array}$ & $\begin{array}{l}232 \\
(4.52 \%)\end{array}$ & $\begin{array}{l}58 \\
(4.53 \%)\end{array}$ & 55 (4.32\%) & 55 (4.25\%) & 64 (4.99\%) & 0.807 \\
\hline $\begin{array}{l}\text { Lipid-lowering } \\
\text { drugs }\end{array}$ & $\begin{array}{l}177 \\
(3.45 \%)\end{array}$ & $\begin{array}{l}40 \\
(3.12 \%)\end{array}$ & 45 (3.54\%) & 48 (3.71\%) & $44(3.43 \%)$ & 0.871 \\
\hline \multicolumn{7}{|c|}{$\begin{array}{l}\text { Abbreviation: PAD, peripheral arterial disease; BMI, body mass index; WC, waist circumference; HC, hip } \\
\text { circumference; SBP, systolic blood pressure; DBP, diastolic blood pressure; CHD, coronary heart } \\
\text { disease; CKD, chronic kidney disease; FBG: fasting blood glucose; Hcy, homocysteine; eGFR, } \\
\text { estimated glomerular filtration rate; HDL-C, high-density lipoprotein cholesterol; TC, total cholesterol; } \\
\text { TG, Triglycerides; TBiL, total bilirubin; ALT, alanine aminotransferase; AST, aspartate } \\
\text { aminotransferase; eGFR, estimated glomerular filtration rate. }\end{array}$} \\
\hline \multicolumn{7}{|c|}{$\begin{array}{l}\text { \$diabetes mellitus was defined as self-reported physician diagnosis of diabetes or FBG concentration } \\
\geq 7.0 \mathrm{mmol} / \mathrm{L} \text { or use of glucose-lowering drugs. }\end{array}$} \\
\hline
\end{tabular}

The multivariate logistic regression model was performed to evaluate the associations between LgTBiL and PAD. The effect values (OR) and 95\% confidence interval (Cl) for full-adjustment were listed in Table 2, per 1 unit of LgTBiL higher were associated with $56 \%$ increased risk of PAD, but the results did not 
reach statistical significance. For sensitivity analysis, we also handled LgTBiL as quartiles and a categorical variable, respectively. Compared with participants in Q2, a higher risk of PAD was found in participants in Q1 (OR,1.89; 95\% Cl: 1.20-2.96), Q3 (OR,1.58; 95\% Cl: 0.98-2.53) and Q4 (OR,2.16; 95\%Cl: 1.35-3.45), respectively. It can be seen from Table 2 that among participants in Q3, the risk of increased PAD was not significant; therefore, Q3 and Q2 populations are combined as a control group. The result showed that in comparison with participants in Q2-Q3 of LgTBiL, there was a significantly increased risk of PAD for participants in both Q1 (OR,1.49; 95\%Cl: 1.04-2.14) and Q4 (OR,1.70; 95\%Cl: 1.16-2.48). The result suggested that the relations between $\mathrm{LgTBiL}$ and PAD were not merely linear.

Table 2

ORs and $95 \% \mathrm{Cl}$ of PAD incidence according to TBiL levels $(\mu \mathrm{mol} / \mathrm{L})$

\begin{tabular}{|c|c|c|c|c|c|}
\hline \multirow[t]{2}{*}{ LgTBiL } & \multirow[t]{2}{*}{$\mathbf{N}$} & \multirow{2}{*}{$\begin{array}{l}\text { Events } \\
\text { (\%) }\end{array}$} & \multicolumn{3}{|c|}{ PAD OR $(95 \% \mathrm{Cl}), P$ value } \\
\hline & & & Crude model & Model 1 & Model 2 \\
\hline Continuous & 5129 & $\begin{array}{l}350 \\
(3.21 \%)\end{array}$ & $\begin{array}{l}0.74(0.33,1.68) \\
0.473\end{array}$ & $\begin{array}{l}1.12(0.48,2.63) \\
0.789\end{array}$ & $\begin{array}{l}1.56(0.64,3.81) \\
0.332\end{array}$ \\
\hline \multicolumn{6}{|l|}{ Quartiles } \\
\hline Q1 (<1.04) & 1281 & $\begin{array}{l}64 \\
(5.00 \%)\end{array}$ & $\begin{array}{l}1.97(1.29,3.03) \\
0.002\end{array}$ & $\begin{array}{l}1.94(1.25,3.02) \\
0.003\end{array}$ & $\begin{array}{l}1.89(1.20,2.96) \\
0.006\end{array}$ \\
\hline $\begin{array}{l}\text { Q2 (1.04- } \\
1.15)\end{array}$ & 1272 & $\begin{array}{l}33 \\
(2.59 \%)\end{array}$ & 1.00 & 1.00 & 1.00 \\
\hline $\begin{array}{l}\text { Q3 (1.15- } \\
1.27)\end{array}$ & 1293 & $\begin{array}{l}45 \\
(3.48 \%)\end{array}$ & $\begin{array}{l}1.35(0.86,2.14) \\
0.193\end{array}$ & $\begin{array}{l}1.54(0.96,2.46) \\
0.071\end{array}$ & $\begin{array}{l}1.58(0.98,2.53) \\
0.059\end{array}$ \\
\hline Q4 ( $\geq 1.27)$ & 1283 & $\begin{array}{l}52 \\
(4.05 \%)\end{array}$ & $\begin{array}{l}1.59(1.02,2.47) \\
0.041\end{array}$ & $\begin{array}{l}1.93(1.22,3.06) \\
0.005\end{array}$ & $\begin{array}{l}2.16(1.35,3.45) \\
0.001\end{array}$ \\
\hline \multicolumn{6}{|l|}{ Categories } \\
\hline Q1 (<1.04) & 1281 & $\begin{array}{l}64 \\
(5.00 \%)\end{array}$ & $\begin{array}{l}1.68(1.20,2.35) \\
0.003\end{array}$ & $\begin{array}{l}1.55(1.09,2.20) \\
0.015\end{array}$ & $\begin{array}{l}1.49(1.04,2.14) \\
0.031\end{array}$ \\
\hline $\begin{array}{l}\text { Q2-Q3 (1.04- } \\
1.27)\end{array}$ & 2565 & $\begin{array}{l}78 \\
(3.04 \%)\end{array}$ & 1.00 & 1.00 & 1.00 \\
\hline Q4 ( $\geq 1.27)$ & 1283 & $\begin{array}{l}52 \\
(4.05 \%)\end{array}$ & $\begin{array}{l}1.35(0.94,1.93) \\
0.103\end{array}$ & $\begin{array}{l}1.53(1.06,2.23) \\
0.024\end{array}$ & $\begin{array}{l}1.70(1.16,2.48) \\
0.007\end{array}$ \\
\hline \multicolumn{6}{|c|}{ Crude model was adjusted for none. } \\
\hline \multicolumn{6}{|c|}{ Model 1 was adjusted for age, BMI, WC, HC, SBP, DBP, pulse, smoking status, drinking status. } \\
\hline \multicolumn{6}{|c|}{$\begin{array}{l}\text { Model } 2 \text { was adjusted for all variables in Model } 1 \text { plus adjusted for FBG, TC, TG, Hcy, pulse, HDL-C, } \\
\text { AST, ALT, antihypertensive drugs, glucose-lowering drugs, lipid-lowering drugs. }\end{array}$} \\
\hline
\end{tabular}


To find the nonlinear relationship between LgTBiL and PAD, we used a generalized additive model and penalized spline method (Fig. 1). The smoothing curve showed that there existed a U-shaped curve association of LgTBiL with PAD in Chinese male adults with hypertension (after adjusting age, BMI, WC, HC, SBP, DBP, pulse, smoking status, alcohol consumption, FBG, TC, TG, Hcy, HDL-C, AST, ALT, eGFR, CHD, antihypertensive drugs, glucose-lowering drugs, lipid-lowering drugs). Furthermore, we further fitted the relationship between LgTBiL and PAD using the two-piecewise logistic regression model (Table 3), and calculated the inflection point was $1.08(\mathrm{LgTBiL}=1.08, \mathrm{TBiL}=12.02 \mu \mathrm{mol} / \mathrm{L})$. Among the participants whose $\mathrm{LgTBiL}<1.08 \mu \mathrm{mol} / \mathrm{L}$ ( TBiL $<12.02 \mu \mathrm{mol} / \mathrm{L}$ ), there was a significant trend towards decreasing risk of PAD development with increment of LgTBiL (OR, $0.11 ; 95 \% \mathrm{Cl}: 0.02-0.83)$. However, the risk of PAD development significantly increased with the increasing level of LgTBiL (OR, 5.26; 95\%Cl: 59-17.38) in participants with $\mathrm{LgTBiL} \geq 1.08$ ( TBiL $\geq 12.02 \mu \mathrm{mol} / \mathrm{L}$ ).

Table 3

Threshold effect analysis of TBil on PAD using two piecewise logistic regression model

\begin{tabular}{|c|c|c|c|c|c|}
\hline \multirow[t]{2}{*}{ LgTBiL } & \multirow[t]{2}{*}{$\mathbf{N}$} & \multirow{2}{*}{$\begin{array}{l}\text { Events } \\
\text { (\%) }\end{array}$} & \multicolumn{3}{|c|}{ PAD OR $(95 \% \mathrm{Cl}) \dagger, P$ value } \\
\hline & & & Crude model & Model 1 & Model 2 \\
\hline Continuous & 5129 & $\begin{array}{l}194 \\
(3.78 \%)\end{array}$ & $\begin{array}{l}0.74(0.33 \\
1.68) 0.473\end{array}$ & $\begin{array}{l}1.12(0.48,2.63) \\
0.789\end{array}$ & $\begin{array}{l}1.56(0.64,3.81) \\
0.332\end{array}$ \\
\hline \multicolumn{6}{|l|}{ Inflection point } \\
\hline$\leq 1.08$ & 1739 & $\begin{array}{l}72 \\
(4.14 \%)\end{array}$ & $\begin{array}{l}0.07(0.01 \\
0.44) 0.004\end{array}$ & $\begin{array}{l}0.10(0.01,0.73) \\
0.023\end{array}$ & $\begin{array}{l}0.11(0.02,0.83) \\
0.032\end{array}$ \\
\hline$>1.08$ & 3390 & $\begin{array}{l}122 \\
(3.59 \%)\end{array}$ & $\begin{array}{l}2.40(0.77 \\
7.51) 0.131\end{array}$ & $\begin{array}{l}3.45(1.08 \\
11.05) 0.037\end{array}$ & $\begin{array}{l}5.26(1.59 \\
17.38) 0.007\end{array}$ \\
\hline $\begin{array}{l}P \text { for log likelihood } \\
\text { ratio test }\end{array}$ & & & 0.009 & 0.012 & 0.007 \\
\hline
\end{tabular}

Crude model was adjusted for none.

Model 1 was adjusted for age, BMI, WC, HC, SBP, DBP, pulse, smoking status, drinking status.

Model 2 was adjusted for all variables in Model 1 plus adjusted for FBG, TC, TG, Hcy, HDL-C, AST, ALT, eGFR, CHD, antihypertensive drugs, glucose-lowering drugs, lipid-lowering drugs.

Figure S1 Flow chart of study participants

\section{Subgroup analyses}

We performed exploratory subgroup analyses to assess the association between LgTBiL and PAD in two groups of participants separated by the turning point of LgTBiL (1.08) (Fig. 2). The effect of LgTBiL on PAD showed no significant difference in the following subgroups: age ( $<65 \mathrm{vs.} \geq 65$ years), BMI ( $<24 \mathrm{vs.}$ $\geq 24 \mathrm{~kg} / \mathrm{m}^{2}$ ), smoking status (never vs. Former vs. current), AST ( $\leq 40 \mathrm{vs.} \geq 40 \mathrm{U} / \mathrm{L}$ ), diabetes mellitus (no vs. yes), CKD ( no vs. yes) in both groups (all $P$ for interactions $>0.05$ ), after adjustment for age, BMI, WC, HC, SBP, DBP, pulse, smoking status, alcohol consumption, FBG, TC, TG, Hcy, HDL-C, AST, ALT, eGFR, 
$\mathrm{CHD}$, antihypertensive drugs, glucose-lowering drugs, lipid-lowering drugs, except for the stratifying variable.

\section{Discussion}

For the first time, we found a independently U-shaped association between TBiL and PAD among hypertensive male subjects, and then revealed a turn point $(\mathrm{LgTBiL}=1.08, \mathrm{TBiL}=12.02 \mu \mathrm{mol} / \mathrm{L})$ by threshold effect analysis.

Several previous studies had examined the relationships between TBiL levels and PAD(17-19). Ozeki et al.(17)reported that serum bilirubin concentration was significantly negatively associated with PAD prevalence in 935 cardiology patients. Lan et al.(18)conducted a cross-section study, which included 543 participants with hypertension (mean age: $62.7 \pm 12.4$ years). The result showed that per 1 unit increment of TBiL was associated with $8.6 \%$ (OR, $0.914 ; 95 \% \mathrm{Cl}$ : 0.845-0.990) lower risk of PAD; besides, an independently negatively relationship between TBiL and PAD (OR,0.884; 95\% Cl: $0.792-0.985)$ was merely found in males but not in females. A cross-sectional examination from the National Health and Nutrition Examination Survey (1999 to 2004) analyzed 7075 adults with data available on the ankle brachial index, serum total bilirubin level, and PAD risk factors. The result showed that a $0.1 \mathrm{mg} / \mathrm{dL}$ increase in bilirubin level was associated with a $6 \%$ reduction in the odds of PAD (OR 0.94 [95\% $\mathrm{Cl} 0.90$ to 0.98]), and this association is more influential in men compared with women(19). However, these studies did not discuss a nonlinear relationship between TBiL and PAD.

In the current study, some new insights were demonstrated in hypertensive males patients. Our in-depth study showed that the association between TBiL and PAD risk was not a simple linear association but a U-shaped curve, suggesting that low and high TBiL levels were associated with increased PAD risk. The reasons for these contradictory findings might be different total serum bilirubin levels, and the distribution of total serum bilirubin levels may vary depending on the race(26, 27), age(28), health $\operatorname{status}(29,30)$, and sample size of the subjects. We conducted a cross-section study included 5,129 Chinese hypertensive males subjects, the mean age of our study participants was $63.86 \pm 9.25$ years, the median serum concentrations of TBiL was $14.10 \mu \mathrm{mol} / \mathrm{L}$ (interquartile range 11.00 to $18.60 \mu \mathrm{mol} / \mathrm{L}$ ); However, Ozeki et al.'s study(17) enrolled 935 Japanese cardiology patients ( median serum bilirubin: about $8.55 \mu \mathrm{mol} / \mathrm{L}$ ), and Lan et al.'s study(18) analyzed 543 Chinese participants with hypertension, the mean serum bilirubin was $12.2 \pm 5.6 \mu \mathrm{mol} / \mathrm{L}$, respectively. At the same time, Perlstein et al.(19)conducted a cross-sectional examination that included 7075 various racial groups adults; the median total bilirubin level was 11.97 (interquartile range 8.55 to 13.68) $\mu \mathrm{mol} / \mathrm{L}$. Due to the small sample size of the above study and the relatively lower bilirubin levels. Thence, we speculate that the negative relationship might be part of the U-shaped curve in this study. Secondly, previous studies were carried out in patients with cardiology and hypertension, and the general people, while the current study conducted in participants with hypertension accompanied by hyper-homocysteinemia (HHcy). About $75 \%$ of hypertension patients in China have hyper-homocysteinemia simultaneously(31), HHcy was defined as Hcy level $\geq$ 
$10 \mu \mathrm{mol} / \mathrm{L}(32)$, TBiL may be heterogeneous for different diseases; the threshold point of injury is different in different diseases.

The current study found that the OR value of Model 1 has changed direction compared with the crude model. The differences in outcomes between the crude model and Model 1 in our study may be explained by the widespread influence of covariates, such as smoking, blood pressure, age, and BMI. Lu et al.(33) conducted a meta-analysis of the association between cigarette smoking and PAD. The result demonstrated that smoking increased risk of PAD. According to the 2017 ESC Guidelines on the Diagnosis and Treatment of Peripheral Arterial Diseases, in collaboration with the European Society for Vascular Surgery, the major risk factors for PAD are smoking, hypertension, diabetes, abnormal lipid metabolism, obesity, etc.(8). Because the effect of these covariates on PAD is too significant to cover up the effect of serum bilirubin on PAD, the independent effect of serum bilirubin on PAD is only reflected after adjusting it.

To our knowledge, TBiL is a potent endogenous antioxidant protecting cells from a 10000 -fold higher concentration of oxidants(34-36); Enhance, lower bilirubin levels could induce the oxidative stress and inflammation which are related to the pathogenesis and development of arteriosclerosis(37). However, the exact mechanisms of excessive TBiL levels with PAD remain unknown. One possible reason could account for the association between excessive TBiL and increased risk of PAD, which was that excessive TBiL such as dominant jaundice might indicate potential liver cell damage, such as hepatocellular or obstructive jaundice, which in turn causes elevated levels of transaminases and alkaline phosphatase(38), the increased levels of transaminase and alkaline phosphatase are associated with an increased risk of $\operatorname{CVD}(20,39)$.

The potential limitations of our study should also be noted. First, we cannot draw any causal relationship between serum bilirubin and PAD from the data because this is a cross-sectional study. Second, the serum bilirubin was only assessed at the baseline in the present study; multiple tests may make the results more accurate. Lastly, this study was conducted in Chinese hypertension male participants, the generalizability of the findings to other populations remains to be determined.

\section{Conclusions}

In summary, this cross-sectional study showed a U-shaped curve for the risk of PAD with TBiL concentration in hypertensive male patients, with a turning point at about $12.02 \mu \mathrm{mol} / \mathrm{L}$. Further welldesigned prospective cohort studies are needed to determine the association's causality and clarify the potential underlying mechanisms of TBiL in PAD.

\section{Declarations}

\section{ACKNOWLEDGEMENTS}


Authors' contributions: YMS participated in the literature search, data analysis, and data interpretation. YMS wrote the manuscript. LHH extracted and collected data. LHH, MHL, XH, CCD, WZ, TW, LJZ conceived of the study and participated in its design and coordination. HHB and XSC participated in the study design and provided critical revision. All authors read and approved the final manuscript.

Funding: This work was supported by the Science and Technology Innovation Platform Project of Jiangxi Province (Grant number: 20165BCD41005) and the Project of Jiangxi Outstanding Person Foundation (Grant number: 20192BCBL23024).

Conflict of Interests: The authors declare that they have no conflict of interest.

Ethical approval: All procedures performed in studies involving human participants were following the ethical standards of the institutional and national research committee and with the 1964 Helsinki declaration and its later amendments or comparable ethical standards.

Informed consent: Informed consent was obtained from all individual participants included in the study.

\section{References}

1. Gerhard-Herman MD. Gornik HL, Barrett C, et al 2016 AHA/ACC Guideline on the Management of Patients With Lower Extremity Peripheral Artery Disease: A Report of the American College of Cardiology/American Heart Association Task Force on Clinical Practice Guidelines. J AM COLL CARDIOL 2017;69:e71-126.

2. Eraso LH. Fukaya E, Mohler ER. Xie D, Sha D. Berger JS. Peripheral arterial disease, prevalence and cumulative risk factor profile analysis. EUR J PREV CARDIOL 2014;21:704 - 11.

3. Wang Z. Chen Z, Zhang L, et al Status of Hypertension in China: Results From the China Hypertension Survey, 2012-2015. CIRCULATION 2018;137:2344-56.

4. Murabito JM. Evans JC, Nieto K. Larson MG, Levy D. Wilson PW. Prevalence and clinical correlates of peripheral arterial disease in the Framingham Offspring Study. AM HEART J 2002;143:961-5.

5. Diehm C. Schuster A, Allenberg JR, et al High prevalence of peripheral arterial disease and comorbidity in 6880 primary care patients: cross-sectional study. ATHEROSCLEROSIS 2004;172:95105.

6. Kannel WB. McGee DL. Update on some epidemiologic features of intermittent claudication: the Framingham Study. J AM GERIATR SOC 1985;33:13 - 8.

7. Schroder F. Diehm N, Kareem S, et al A modified calculation of ankle-brachial pressure index is far more sensitive in the detection of peripheral arterial disease. J VASC SURG 2006;44:531-6.

8. Halliday A. Bax JJ. The 2017 ESC Guidelines on the Diagnosis and Treatment of Peripheral Arterial Diseases, in Collaboration With the European Society for Vascular Surgery (ESVS). Eur J Vasc Endovasc Surg 2018;55:301-2. 
9. Lin JP. O'Donnell CJ, Schwaiger JP, et al Association between the UGT1A1*28 allele, bilirubin levels, and coronary heart disease in the Framingham Heart Study. CIRCULATION 2006;114:1476-81.

10. Breimer LH. Wannamethee G, Ebrahim S. Shaper AG. Serum bilirubin and risk of ischemic heart disease in middle-aged British men. CLIN CHEM 1995;41:1504-8.

11. Lapenna D. Ciofani G, Pierdomenico SD. Giamberardino MA, Ucchino S. Davi G. Association of serum bilirubin with oxidant damage of human atherosclerotic plaques and the severity of atherosclerosis. CLIN EXP MED 2018;18:119 - 24.

12. Huang YH. Yang YC, Lu FH. Sun ZJ, Wu JS. Chang CJ. Serum Bilirubin Is Inversely Associated with Increased Arterial Stiffness in Men with Pre-Hypertension but Not Normotension. PLOS ONE 2016;11:e146226.

13. Stocker R. Yamamoto Y, McDonagh AF. Glazer AN, Ames BN. Bilirubin is an antioxidant of possible physiological importance. SCIENCE 1987;235:1043-6.

14. Schwertner HA. Jackson WG, Tolan G. Association of low serum concentration of bilirubin with increased risk of coronary artery disease. CLIN CHEM 1994;40:18-23.

15. 15.

16. Akboga MK. Canpolat U, Sahinarslan A, et al Association of serum total bilirubin level with severity of coronary atherosclerosis is linked to systemic inflammation. ATHEROSCLEROSIS 2015;240:110-4.

17. Ozeki M. Morita $\mathrm{H}$, Miyamura $\mathrm{M}$, et al High serum bilirubin is associated with lower prevalence of peripheral arterial disease among cardiac patients. CLIN CHIM ACTA 2018;476:60 - 6.

18. Lan Y. Liu H, Liu J. Zhao H, Wang H. The Relationship Between Serum Bilirubin Levels and Peripheral Arterial Disease and Gender Difference in Patients With Hypertension: BEST Study. ANGIOLOGY 2020;71:340-8.

19. Perlstein TS. Pande RL, Beckman JA. Creager MA. Serum total bilirubin level and prevalent lowerextremity peripheral arterial disease: National Health and Nutrition Examination Survey (NHANES) 1999 to 2004. Arterioscler Thromb Vasc Biol 2008;28:166 - 72.

20. Kunutsor SK. Apekey TA, Khan H. Liver enzymes and risk of cardiovascular disease in the general population: a meta-analysis of prospective cohort studies. ATHEROSCLEROSIS 2014;236:7-17.

21. Walden CE. Knopp RH, Johnson JL. Heiss G, Wahl PW. Hoover JJ. Effect of estrogen/progestin potency on clinical chemistry measures. The Lipid Research Clinics Program Prevalence Study. AM J EPIDEMIOL 1986;123:517 - 31.

22. Yu Y. Hu L, Huang X. Zhou W, Bao H. Cheng X. BMI modifies the association between serum HDL cholesterol and stroke in a hypertensive population without atrial fibrillation. J ENDOCRINOL INVEST 2020.

23. Levey AS. Stevens LA, Schmid $\mathrm{CH}$, et al A new equation to estimate glomerular filtration rate. ANN INTERN MED 2009;150:604 - 12.

24. Aboyans V. Criqui MH, Abraham P, et al Measurement and interpretation of the ankle-brachial index: a scientific statement from the American Heart Association. CIRCULATION 2012;126:2890 - 909. 
25. Hu L. Huang X, Zhou W, et al Associations between resting heart rate, hypertension, and stroke: A population-based cross-sectional study. The Journal of Clinical Hypertension 2019;21:589 - 97.

26. Jung $\mathrm{CH}$. Lee $\mathrm{MJ}$, Kang $\mathrm{YM}$, et al Higher serum bilirubin level as a protective factor for the development of diabetes in healthy Korean men: a 4 year retrospective longitudinal study. METABOLISM 2014;63:87-93.

27. Oda E. Kawai R. A possible cross-sectional association of serum total bilirubin with coronary heart disease and stroke in a Japanese health screening population. HEART VESSELS 2012;27:29-36.

28. Boland BS. Dong MH, Bettencourt R. Barrett-Connor E, Loomba R. Association of serum bilirubin with aging and mortality. J Clin Exp Hepatol 2014;4:1-7.

29. 29.

30. Chen YH. Hung SC, Tarng DC. Serum bilirubin links UGT1A1*28 polymorphism and predicts longterm cardiovascular events and mortality in chronic hemodialysis patients. Clin J Am Soc Nephrol $2011 ; 6: 567-74$.

31. Zhang Q. Qiu DX, Fu RL, et al H-Type Hypertension and C Reactive Protein in Recurrence of Ischemic Stroke. Int J Environ Res Public Health 2016;13.

32. Qin X. Huo Y. H-Type hypertension, stroke and diabetes in China: Opportunities for primary prevention. J DIABETES 2016;8:38-40.

33. Lu L. Mackay DF, Pell JP. Meta-analysis of the association between cigarette smoking and peripheral arterial disease. HEART 2014;100:414 - 23.

34. Akboga MK. Canpolat U, Sahinarslan A, et al Association of serum total bilirubin level with severity of coronary atherosclerosis is linked to systemic inflammation. ATHEROSCLEROSIS 2015;240:110-4.

35. Breimer LH. Spyropolous KA, Winder AF. Mikhailidis DP, Hamilton G. Is bilirubin protective against coronary artery disease? CLIN CHEM 1994;40:1987-8.

36. Baranano DE. Rao M, Ferris CD. Snyder SH. Biliverdin reductase: a major physiologic cytoprotectant. Proc Natl Acad Sci U S A 2002;99:16093-8.

37. Leopold JA. Loscalzo J. Oxidative enzymopathies and vascular disease. Arterioscler Thromb Vasc Biol 2005;25:1332-40.

38. Franchini M. Targher G, Lippi G. Serum bilirubin levels and cardiovascular disease risk: a Janus Bifrons? ADV CLIN CHEM 2010;50:47-63.

39. Schindhelm RK. Dekker JM, Nijpels G, et al Alanine aminotransferase predicts coronary heart disease events: a 10-year follow-up of the Hoorn Study. ATHEROSCLEROSIS 2007;191:391-6.

\section{Figures}




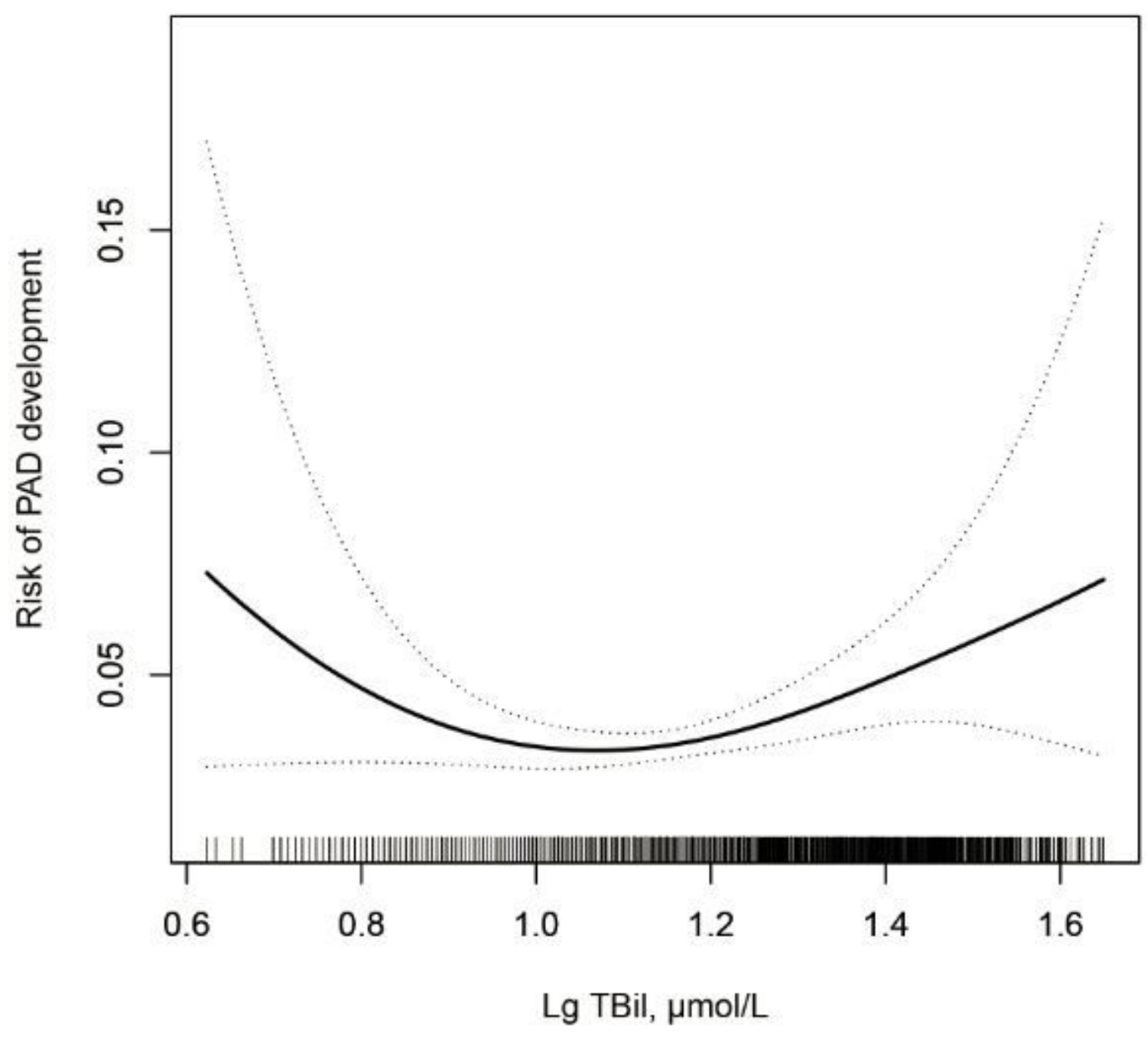

Figure 1

Association between LgTBil and the prevalence of PAD A nonlinear association between TBil and the prevalence of PAD was found $(P<0.05)$. The solid line and dashed line represent the estimated values and their corresponding $95 \%$ confidence interval. Adjustment factors included age, BMI, WC, HC, SBP, DBP, pulse, smoking status, drinking status, FBG, TC, TG, Hcy, HDL-C, AST, ALT, eGFR, CHD, antihypertensive drugs, glucose-lowering drugs, lipid-lowering drugs. 


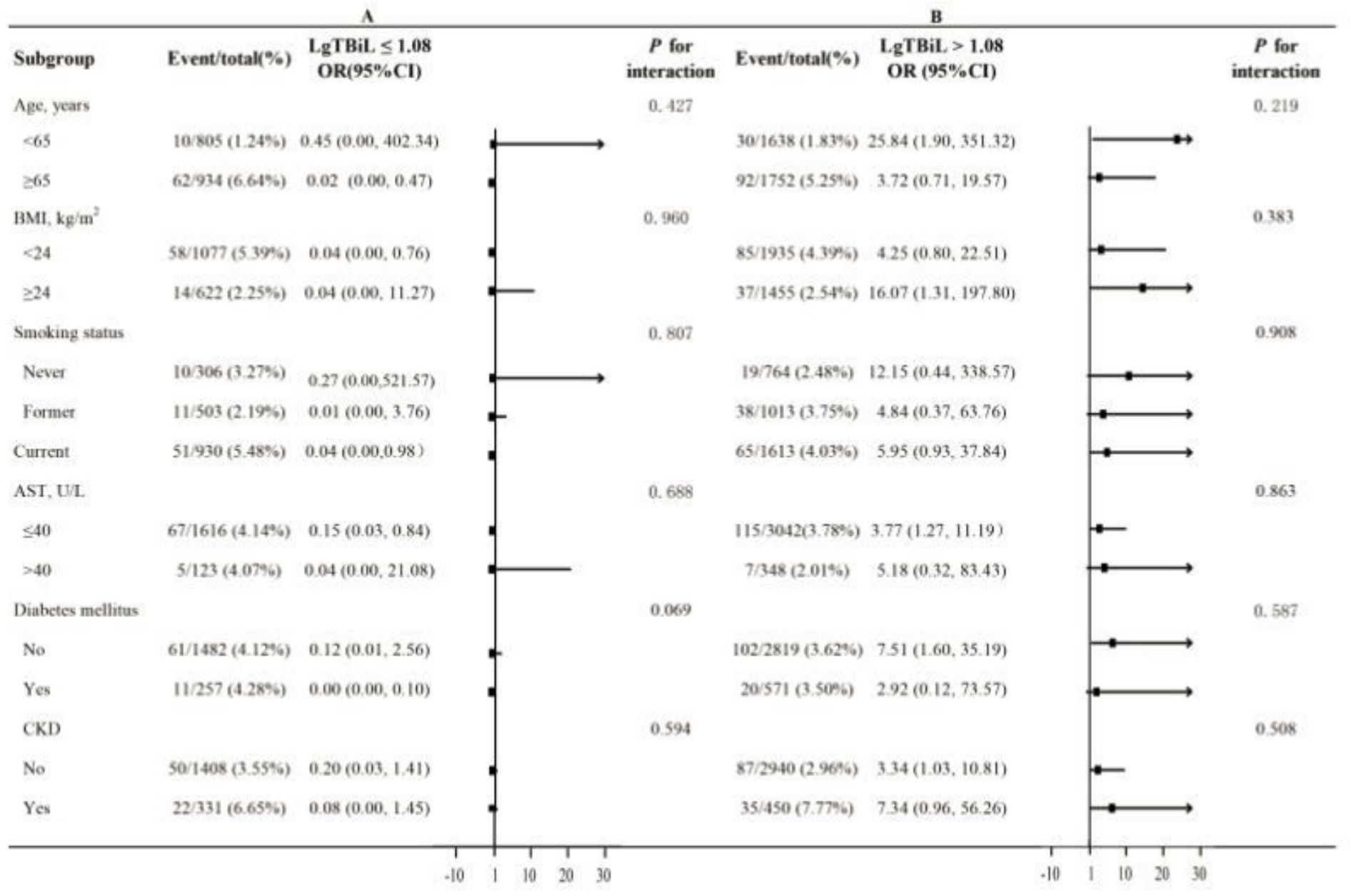

\section{Figure 2}

Stratified analysis for the PAD and LgTBil in various subgroups divided by 1.08. (A: LgTBil $\leq 1.08$ $\mu \mathrm{mol} / \mathrm{IL}, \mathrm{B}$ : LgTBil >1.08 $\mu \mathrm{mol} / \mathrm{lL}$ ). *Each subgroup analysis adjusted for age, BMI, WC, HC, SBP, DBP, pulse, smoking status, drinking status, FBG, TC, TG, Hcy, HDL-C, AST, ALT, eGFR, CHD, antihypertensive drugs, glucose-lowering drugs, lipid-lowering drugs囚except for the stratifying variable.

\section{Supplementary Files}

This is a list of supplementary files associated with this preprint. Click to download.

- FigureS1.tif 